\title{
A METHOD FOR ESTIMATING PARALLAXES OF VCBS: MODIFICATION TO HIPPARCOS PARALLAX MEASUREMENTS
}

\author{
M. A. Al-WARDAT*,,$\uparrow$, A. TAANI ${ }^{\ddagger}$ and M. ASPLUND ${ }^{\S}$ \\ *Department of Physics, Al-Hussein Bin Talal University \\ P.O.Box 20, 71111, Ma'an, Jordan \\ ${ }^{\dagger}$ Sabbatical visitor, Physics Department, Yarmouk University \\ P.O.B. 566 Irbid, 21163 Jordan \\ $¥$ National Astronomical Observatories \\ Chinese Academy of Sciences, Beijing 100012, China \\ $\S$ Max Planck Institute for Astrophysics \\ Postfach 1317, 85741 Garching, Germany \\ 『mwardat@ahu.edu.jo
}

\begin{abstract}
We presented a method useful for estimating parallaxes of visually close binary stars (VCBS). The method depends on atmospheric modeling of the components of the VCBS. We construct model atmospheres by using a grid of Kurucz solar metalicity blanketed models ${ }^{1}$, which can be applied to calculate the synthetic spectral energy distribution for each component of the binary separately. In addition to study the entire system. However, the entire observational spectral energy distribution of the system was used as a reference for the comparison with the synthetic ones. We choose Hip4809 system to investigate the method, and we also study its physical and geometrical parameters. The new parallax of the system was estimated as $\pi=40.32 \pm 5.00$ mas, which disagrees with the Hipparcos parallax measurement $(13.94 \pm 0.90$ mas). Furthermore the model atmosphere parameters of the components of the system were derived as: $T_{\text {eff }}^{a}=5625 \pm$ $75 \mathrm{~K}, T_{\text {eff }}^{b}=5575 \pm 75 \mathrm{~K}, \log g_{a}=4.50 \pm 0.13, \log g_{b}=4.50 \pm 0.13, R_{a}=0.94 \pm 0.12 R_{\odot}$, $R_{b}=0.93 \pm 0.12 R_{\odot}$, Depending on the derived parameters, the masses of the system's components were estimated as $1.03 \pm 0.02 M_{\odot}$ and $1.01 \pm 0.02 M_{\odot}$ for the primary and secondary components respectively, and their spectral types were concluded as G6V for both of them.
\end{abstract}

Keywords: Stars: fundamental parameters — stars: binaries: visual — stars: atmospheres modeling — stars: individual: Hip4809.

\section{Introduction}

The study of binary systems, in general, plays an important role in determining several key stellar parameters (i.e. to measure accurate the stellar masses, to test the evolutionary models and the star formation theories), which is more complicated in the case of visually close binary stars (VCBS). Although hundreds of binary systems with periods in the order of 10 years or less, are routinely observed by different groups of high resolution techniques around the world, but there is still a lake 
Table 1. Data from SIMBAD.

\begin{tabular}{cc}
\hline & Hip4809 \\
\hline$\alpha_{2000}$ & $01^{h} 01^{m} 43^{s} 581$ \\
$\delta_{2000}$ & $+25 \circ 17^{\prime} 31 .^{\prime \prime} 99$ \\
Tyc & $1743-1174-1$ \\
HD & 6009 \\
Spectral type & G8IV \\
\hline
\end{tabular}

Table 2. Data from Hipparcos and Tycho Catalogues.

\begin{tabular}{cc}
\hline & Hip4809 \\
& HD6009 \\
\hline$V_{J}($ Hip $)$ & $6 \cdot^{m} 71$ \\
$B_{T}$ & $7 \cdot^{m} 683 \pm 0.008$ \\
$V_{T}$ & $6 .^{m} 806 \pm 0.006$ \\
$(B-V)_{J}($ Tycho $)$ & $0 .^{m} 783 \pm 0.007$ \\
Hip Trig. Parallax (mas) & $13.94 \pm 0.90$ \\
Tyc Trig. Parallax (mas) & $12.3 \pm 5.3$ \\
\hline
\end{tabular}

in the determination of the individual physical parameters of the systems' components. However, a variety of observational techniques have been brought to bear in searches for VCBS. At present, only spectrophotometry and atmospheres modelling came with a complementary solution for this problem, by giving an accurate determination of the effective temperature, radius, spectral type and luminosity class for each component of a binary system. The method was successfully applied to some binary systems like ADS11061, Cou1289, Cou1291, Hip11352 and Hip11253. ${ }^{2-5}$ In this work, we presented here a method for determination of systems parameters from estimation of the trigonometric parallaxes of VCBS. In order to have a clear image, we shall examine the parallax of the binary system Hip4809 (HD6009). Table 1 contains the SIMBAD data of the system, and Table 2 contains data from Hipparcos and Tycho Catalogues. ${ }^{6}$

Hip4809 is a well known speckle interferometric system, which was observed by different groups of SI around the world. As asuch, it fulfils the requirements to be analyzed by the aforementioned method, and consequently to help in understanding the formation and evolution mechanisms of binary stellar systems.

\section{Atmospheric Modelling}

\subsection{Observational reference spectrum}

Figure 1 shows the observational spectral energy distributions (SED) of the system, taken from. ${ }^{7}$ Note that some of the strong lines and depressions, especially in the red part of the spectrum (around $\lambda 6867 \AA, \lambda 7200 \AA$, and $\lambda 7605 \AA$ ), are $\mathrm{H}_{2} \mathrm{O}$ and $\mathrm{O}_{2}$ telluric lines and depressions. 


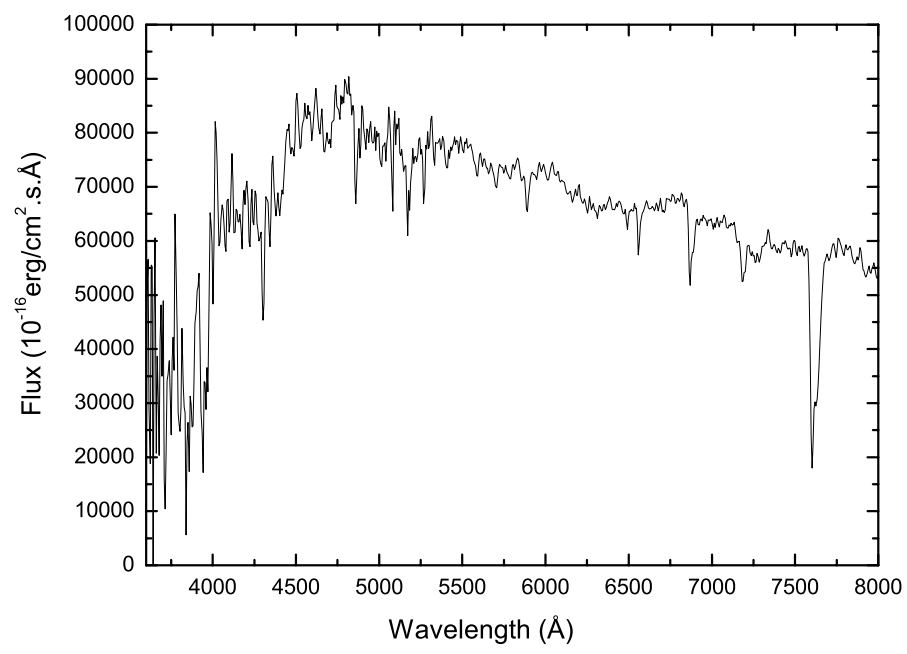

Fig. 1. Spectral energy distribution of the system Hip $4809 .^{7}$

Table 3 lists the synthetic Jonson, Strömgren and Tycho magnitudes and color indices, which were calculated by, ${ }^{7,8}$ depending on the former observational SED and using the passbands and zero points of Refs. 9-12.

\subsection{Input parameters for model atmospheres}

Using $m_{v}=6^{m} 74$ from the previous data and $\triangle m=0^{m} 14$ as the average of all $\triangle m$ measurements under the filter $545 \mathrm{~nm} / 30$ (see table 4 ), which also equal the average of all $\triangle m$ measurements under all filters excluding Hipparcos' one, we can calculate a preliminary individual $m_{v}$ for each component as: $m_{v a}=7^{m} 45$ and $m_{v b}=7^{m} 59$.

Assuming that our stars are main sequence stars depending on the shape of their entire spectrum, we can relay on the main sequence relations and tables ${ }^{13,14}$ to calculate the preliminary parameters of the individual components as follows:

These visual magnitudes along with the system's parallax from Hipparcos catalogue $(\pi=13.94 \pm 0.90, d=71.74 p c)$, and using the following equation:

$$
M_{v}=m_{v}+5-5 \log (d)-A,
$$

give the absolute individual magnitudes as:

$M_{v}^{a}=3^{m} 00$ and $M_{v}^{b}=3^{m} 14$.

When combining these magnitudes with the bolometric corrections from, ${ }^{13,14}$ we get the individual bolometric magnitudes as:

$$
M_{b o l}^{a}=2^{m} 09 \text { and } M_{b o l}^{b}=3^{m} 04,
$$


Table 3. Jonson, Strömgren and Tycho magnitudes and color indices of the entire system. ${ }^{7,8}$

\begin{tabular}{l|c}
\hline & Hip4809 \\
\hline$B_{J}$ & $7^{m} 43 \pm 0.06$ \\
$R_{J}$ & $6^{m} 32 \pm 0.07$ \\
$V_{J}$ & $6^{m} 74 \pm 0.06$ \\
$(B-V)_{J}$ & $0^{m} 67 \pm 0.08$ \\
$v$ & $7^{m} 85 \pm 0.06$ \\
$b$ & $7^{m} 145 \pm 0.06$ \\
$y$ & $6^{m} 70 \pm 0.06$ \\
$v-b$ & $0^{m} 72 \pm 0.08$ \\
$b-y$ & $0^{m} 44 \pm 0.08$ \\
$B_{T}$ & $7^{m} 64 \pm 0.06$ \\
$V_{T}$ & $6^{m} 81 \pm 0.06$ \\
$(B-V)_{T}$ & $0^{m} 82 \pm 0.08$ \\
\hline
\end{tabular}

hence the individual luminosities follow as:

$$
L_{a}=11.59 \pm 1.20 L_{\odot} \text { and } L_{b}=4.83 \pm 0.50 L_{\odot} .
$$

Using the effective temperatures and masses

$$
\begin{gathered}
T_{\mathrm{eff}}^{a}=6900 \mathrm{~K}, \mathrm{~T}_{\mathrm{eff}}^{\mathrm{b}}=6800 \mathrm{~K} \\
M_{a}=1.56 M_{\odot}, M_{b}=1.50 M_{\odot}
\end{gathered}
$$

from the mentioned tables of ${ }^{14}$ we calculate the radii and gravity acceleration (log $g$ ) of the components using the empirical $T_{\text {eff }}-M_{b o l}$ and $S p-M$ relations of main sequence stars:

$$
\begin{array}{r}
\log \left(R / R_{\odot}\right)=0.5 \log \left(L / L_{\odot}\right)-2 \log \left(T / T_{\odot}\right), \\
\log g=\log \left(M / M_{\odot}\right)-2 \log \left(R / R_{\odot}\right)+4.43
\end{array}
$$

as: $R_{a}=1.48 R_{\odot}, R_{b}=1.47 R_{\odot}, \log g_{a}=4.30$ and $\log g_{b}=4 . .30$, where $T_{\odot}=5777 \mathrm{~K}$ was used.

\subsection{Synthetic spectra}

The upper calculated input parameters allowed construction of the model atmospheres of each component using grids of blanketed models (ATLAS9), ${ }^{1}$ where we used solar abundance model atmospheres. Hence the spectral energy distributions in the continuous spectrum for each component were computed.

The total energy flux from a binary star is created from the net luminosity of the components $a$ and $b$ located at a distance $d$ from the Earth. So we can write:

$$
F_{\lambda} \cdot d^{2}=H_{\lambda}^{a} \cdot R_{a}^{2}+H_{\lambda}^{b} \cdot R_{b}^{2}
$$


Table 4. Magnitude difference between the components of the system, along with filter used to obtain the observations.

\begin{tabular}{l|cc}
\hline$\triangle m$ & filter $(\lambda / \Delta \lambda)$ & ref. \\
\hline $0^{m} 28 \pm 0.30$ & $V_{H p}$ & Ref. 6 \\
$0^{m} 17 \pm 0.15$ & $545 \mathrm{~nm} / 30$ & Ref. 15 \\
$0^{m} 30 \pm 0.15$ & $545 \mathrm{~nm} / 30$ & Ref. 15 \\
$0^{m} 19 \pm 0.04$ & $610 \mathrm{~nm} / 20$ & Ref. 16 \\
$0^{m} 10 \pm 0.24$ & $545 \mathrm{~nm} / 30$ & Ref. 17 \\
$0^{m} 12 \pm 0.19$ & $800 \mathrm{~nm} / 110$ & Ref. 18 \\
$0^{m} 16 \pm 0.05$ & $600 \mathrm{~nm} / 30$ & Ref. 18 \\
$0^{m} 00 \pm 0.12$ & $545 \mathrm{~nm} / 30$ & Ref. 18 \\
$0^{m} 00 \pm 0.12$ & $600 \mathrm{~nm} / 30$ & Ref. 18 \\
$0^{m} 21 \pm 0.04$ & $600 \mathrm{~nm} / 30$ & Ref. 19 \\
\hline
\end{tabular}

from which

$$
F_{\lambda}=\left(R_{a}^{2} / d\right)^{2}\left(H_{\lambda}^{a}+H_{\lambda}^{b} \cdot\left(R_{b} / R_{a}\right)^{2}\right),
$$

where $H_{\lambda}^{a}$ and $H_{\lambda}^{b}$ are the fluxes from a unit surface of the corresponding component. $F_{\lambda}$ here represents the entire SED of the system.

Within the criteria of the best fit, which are the maximum values of the absolute flux, the inclination of the spectra, and the profiles of the absorption lines, and starting with the calculated parameters:

$$
\begin{aligned}
& T_{\mathrm{eff}}^{a}=6900 \mathrm{~K}, T_{\mathrm{eff}}^{b}=6800 \mathrm{~K}, \\
& \log g_{a}=4.30, \log g_{b}=4.30, \\
& R_{a}=1.48 R_{\odot}, R_{b}=1.47 R_{\odot},
\end{aligned}
$$

and $d=71.74 \mathrm{pc}$, many attempts were made to achieve the best fit between the observed flux and the total computed one using the iteration method of different sets of parameters. But in all attempts, there was a disagreement in the inclination and absolute flux between the synthetic light curves and the observational one.

The disagreement in the inclination shows that the effective temperatures of the components should be less than those calculated above as a preliminary ones, were we overcame this problem by reducing the effective temperatures (keeping in mind the magnitude difference) tell we achieved the inclination fitness. While the disagreement in the maximum values of the flux refers to a miss-estimation either in the radii of the components or in the parallax of the system. Which means that changing the parallax of the system, affects highly the values of the components' radii, a factor raised up their error values.

Looking back to the calculated $M_{v}$ values using 1, we can see that they disagree with the properties of such kind of stars like $T_{\text {eff }}$ and $B-V$, and the only suspected element in the equation is $d$, the distance of the system. Knowing that the Hipparcos parallax measurements of binary and multiple systems are, in some cases, distorted by the the orbital motion of the components of such systems as noted by, ${ }^{20}$ puts the 
parallax subject to change instead of a postulated one. This is in addition to the aforementioned miss-estimated effective temperatures using Hipparcos parallax.

The attempts included also changing the above calculated first step parameters, by trying different values of $m_{v}, \triangle m$ and parallax from different sources within the error values.

So, hundreds of models were built and compared with the observational SED tell the best fit was achieved using the following set of parameters (Fig. 2):

$$
\begin{gathered}
T_{\mathrm{eff}}^{a}=5625 \pm 75 \mathrm{~K}, T_{\mathrm{eff}}^{b}=5575 \pm 75 \mathrm{~K}, \\
\log g_{a}=4.50 \pm 0.13, \log g_{b}=4.50 \pm 0.13, \\
R_{a}=0.94 \pm 0.12 R_{\odot}, R_{b}=0.93 \pm 0.12 R_{\odot},
\end{gathered}
$$

and $d=24.80 \pm 3.20 \mathrm{pc}$. Thus the luminosities follow as: $L_{a}=0.79 \pm 0.10 L_{\odot}$, and $L_{b}=0.75 \pm 0.10 L_{\odot}$, which lie outside the error of the values calculated above, we shall discus this point later.

The values of the best fit represent adequately enough the parameters of the systems' components. Depending on the tables of ${ }^{14}$ or using by ${ }^{13} S p-T_{\text {eff }}$ empirical relation, the spectral types of the system's components can be estimated as G6 for both of them.

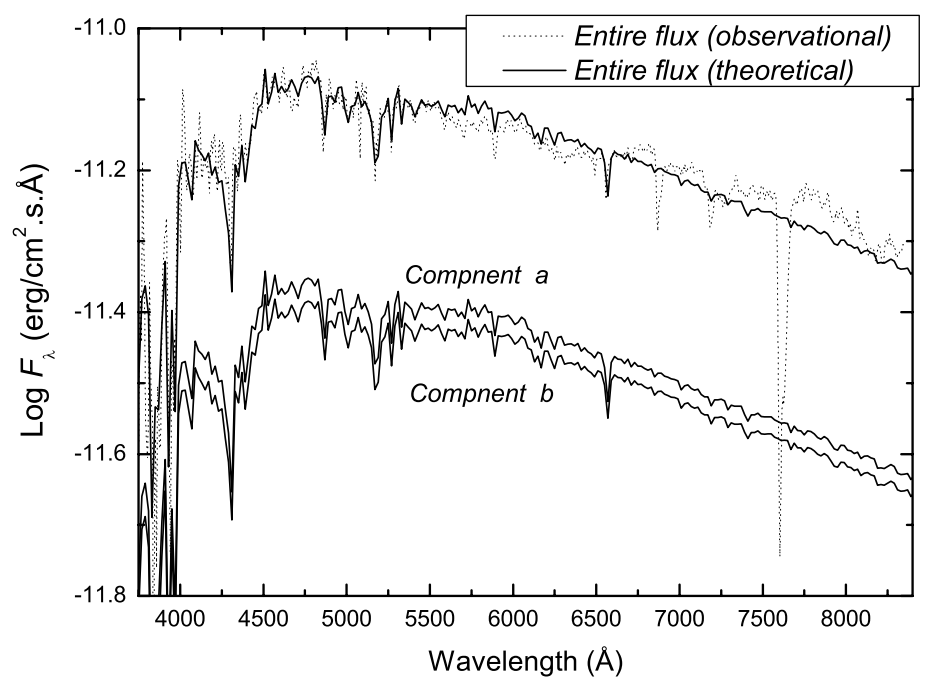

Fig. 2. Dotted line: the entire observational SED in the continuous spectrum of the system. Solid lines: the entire computed SED of the two components, the computed flux of the primary component with $T_{\text {eff }}=5625 \pm 75 \mathrm{~K}, \log g=4.50 \pm 0.13, R=0.94 \pm 0.12 R_{\odot}$, and the computed flux of the secondary component with $T_{\text {eff }}=5575 \pm 75 \mathrm{~K}, \log g=4.50 \pm 0.13, R=0.93 \pm 0.12 R_{\odot}$, and $d=24.80 \pm 3.20 \mathrm{pc}$. 
Table 5. Magnitudes and color indices of the synthetic spectra of the system.

\begin{tabular}{lcccc}
\hline Sys. & Fil. & entire & comp. a & comp. b \\
\hline Johcon & $U$ & $7.77 \pm 0.03$ & 8.46 & 8.58 \\
& $B$ & 7.47 & 8.18 & 8.27 \\
& $V$ & 6.72 & 7.43 & 7.51 \\
& $R$ & 6.32 & 7.04 & 7.10 \\
& $U-B$ & 0.30 & 0.29 & 0.31 \\
& $B-V$ & 0.75 & 0.74 & 0.76 \\
& $V-R$ & 0.40 & 0.40 & 0.41 \\
\hline \multirow{2}{*}{ Ström. } & $u$ & 8.91 & 9.60 & 9.72 \\
& $v$ & 7.87 & 8.57 & 8.67 \\
& $b$ & 7.13 & 7.84 & 7.92 \\
& $y$ & 6.68 & 7.40 & 7.47 \\
& $u-v$ & 1.04 & 1.03 & 1.05 \\
& $v-b$ & 0.74 & 0.73 & 0.75 \\
& $b-y$ & 0.44 & 0.44 & 0.45 \\
\hline \multirow{2}{*}{ Tycho } & $B_{T}$ & 7.66 & 8.37 & 8.47 \\
& $V_{T}$ & 6.80 & 7.51 & 7.59 \\
& $B_{T}-V_{T}$ & 0.87 & 0.86 & 0.88 \\
\hline
\end{tabular}

\section{Synthetic Photometry}

In addition to the direct comparison, we can check reliability of our method of estimating the physical and geometrical parameters by comparing the observed magnitudes of the entire system from different ground or space based telescopes with the entire synthetic ones. For that, we used the following relation ${ }^{10,12}$ :

$$
m_{p}\left[F_{\lambda, s}(\lambda)\right]=-2.5 \log \frac{\int P_{p}(\lambda) F_{\lambda, s}(\lambda) \lambda \mathrm{d} \lambda}{\int P_{p}(\lambda) F_{\lambda, r}(\lambda) \lambda \mathrm{d} \lambda}+\mathrm{ZP}_{p},
$$

to calculate the entire and individual synthetic magnitudes of the system, where $m_{p}$ is the synthetic magnitude of the passband $p, P_{p}(\lambda)$ is the dimensionless sensitivity function of the passband $p, F_{\lambda, s}(\lambda)$ is the synthetic SED of the object and $F_{\lambda, r}(\lambda)$ is the SED of the reference star (Vega). Zero points $\left(\mathrm{ZP}_{p}\right)$ from ${ }^{12}$ (and references there in) were adopted.

The results of the calculated magnitudes and color indices of the entire system and individual components, in different photometrical systems, are shown in Table 5 .

\section{Results and Discussion}

A comparison between the synthetic magnitudes and colors (Table 5) with the observational ones (Tables 2 and 3) shows a very good consistency within three photometrical systems Johnson-Cousins, Strömgren and Tycho. This gives a good indication for the reliability of the estimated parameters of the individual components of the system, which are listed in Table 6 , and proves that our estimated Trigonometric parallax of the system $(\pi=40.32 \pm 5.00 \mathrm{mas})$ is more accurate than those of Hipparcos (13.94 $\pm 0.90 \mathrm{mas})$, and Tycho (12.3 $\pm 5.3 \mathrm{mas})$. 


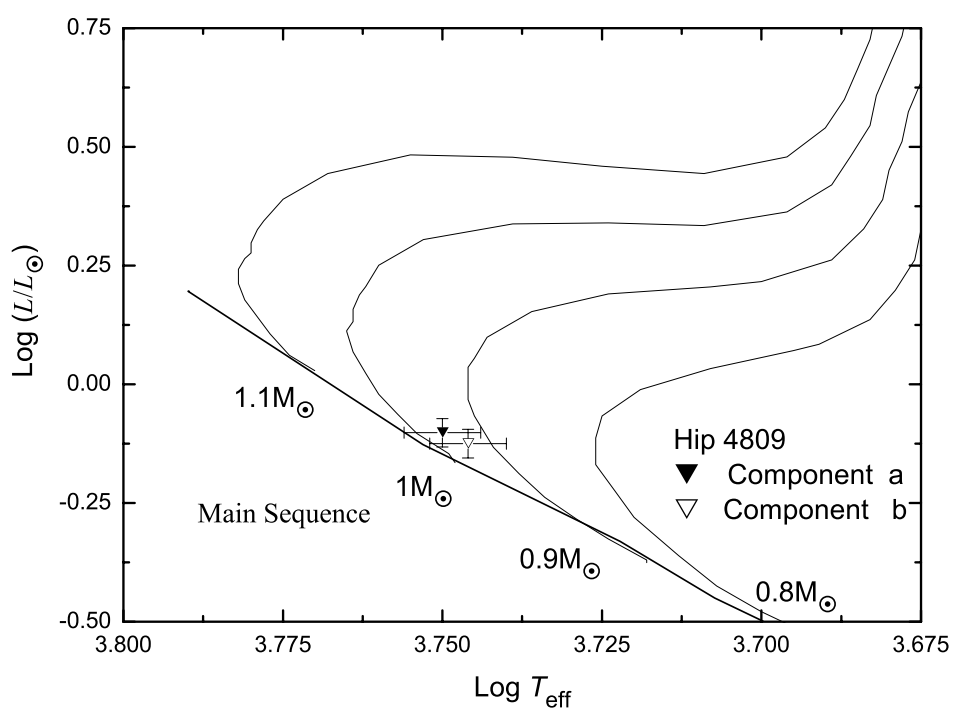

Fig. 3. The system's components on the evolutionary tracks of Ref. 21.

Table 6. Parameters of the system's components.

\begin{tabular}{l|c|c}
\hline Component & $\mathrm{a}$ & $\mathrm{b}$ \\
\hline$T_{\text {eff }}(\mathrm{K})$ & $5625 \pm 75$ & $5575 \pm 75$ \\
Radius $\left(\mathrm{R}_{\odot}\right)$ & $0.94 \pm 0.12$ & $0.93 \pm 0.12$ \\
$\log g$ & $4.50 \pm 0.13$ & $4.50 \pm 0.13$ \\
$L\left(L_{\odot}\right)$ & $0.79 \pm 0.10$ & $0.75 \pm 0.10$ \\
Mass, $\left(M_{\odot}\right)^{*}$ & $1.03 \pm 0.02$ & $1.01 \pm 0.02$ \\
Sp. Type* & $\mathrm{G} 6$ & $\mathrm{G} 6$ \\
\hline Parallax (mas) & \multicolumn{2}{|c}{$40.32 \pm 5.00$} \\
Age (Gy) & \multicolumn{2}{|c}{$2.7 \pm 0.3$} \\
\hline *depending on the tables of. ${ }^{14}$ & \multicolumn{2}{|l}{}
\end{tabular}

Figure 3 shows the positions of the components on the evolutionary tracks of, ${ }^{21}$ where the error bars in the figure include the effect of the parallax uncertainty. The age of the system can be established from the evolutionary tracks as almost as 2.7 Gy.

It is clear from the parameters of the system's components and their positions on the evolutionary tracks that they are very similar solar type main sequence stars, in the early stages of their life. Hence, we can conclude, depending on the formation theories, that the fragmentation is the most likely process for the formation of such 
system. Where ${ }^{22}$ concludes that fragmentation of rotating disk around an incipient central protostar is possible, as long as there is continuing infall, and ${ }^{23}$ pointed out that hierarchical fragmentation during rotational collapse has been invoked to produce binaries and multiple systems.

\section{Conclusions}

On the basis of analyzing the binary system Hip4809 using the complex method of studying VCBS, the following main conclusions can be drawn.

(1) The parameters of the system's components were estimated depending on the best fit between the observational SED and synthetic ones built using the atmospheric modeling of the individual components.

(2) More accurate value of the trigonometric parallax of the system was estimated, where it showed a disagreement with Hipparcos and Tycho measurement.

(3) Depending on the parameters of the system's components and their positions on the evolutionary tracks, we showed that the components are very similar solar type G6 main sequence stars, in the early stages of their life.

(4) The entire and individual $U B V R$ Johnson-Cousins, uvby Strömgren and $B V$ Tycho synthetic magnitudes and colors of the system were calculated.

(5) Finally, the fragmentation was proposed as the most likely process for the formation and evolution of the system.

\section{Acknowledgments}

This work was done during the research visit of the first author to Max Plank Institute for Astrophysics-Garching which was funded by DFG. This work made use of SAO/NASA, SIMBAD, IPAC data systems and CHORIZOS code of photometric and spectrophotometric data analysis.

\section{References}

1. R. L. Kurucz CD-ROM 1, Atomic Data for Opacity Calculations Cambridge: Smithsonian Astrophys (1994).

2. M. A. Al-Wardat $B S A O \mathbf{5 3}$ (2002a) 51.

3. M. A. Al-Wardat $A N \mathbf{3 2 8}$ (2007) 63.

4. M. A. Al-Wardat $A N 330$ (2009a) 385.

5. M. A. Al-Wardat \& H. Widyan Ast.Bu. 64 (2009b) 365.

6. ESA VizieR Online Data Catalog 1239 (1997).

7. M. A. Al-Wardat BSAO 53 (2002b) 58.

8. M. A. Al-Wardat Ast.Bu. 63 (2008) 361.

9. R. C. Bohlin \& R. L. Gilliland AJ , 127 (2004) 3508.

10. J, Maíz Apellániz AJ 131 (2006) 1184.

11. J. B. Holberg, \& P. Bergeron $A J 132$ (2006) 1221.

12. J, Maíz Apellániz ASP Conf. Ser. 364 (2007) 227.

13. K. R. Lang Astrophysical Data I. Planets and Stars New York: Springer (1992). 
14. D. F. Gray The Observation and Analysis of Stellar Photospheres 3rd ed. (Cambridge Univ. Press) 450 (2005).

15. I. I. Balega et al., A\&GA 385 (2002) 87.

16. I. I. Balega et al., $A \mathscr{E} A 422$ (2004) 627.

17. E. A. Pluzhnik $A \mathscr{E} A 431$ (2005) 587.

18. I. I. Balega et al., BSAO 59 (2006) 20.

19. I. I. Balega et al., BSAO 62 (2007) 339.

20. N. I. Shatskii \& A. A. Tokovinin Ast.L 24 (1998) 673.

21. L. Girardi et al., A\&A. Suppl. 141 (2000) 371.

22. I. A. Bonnell MNRAS 269 (1994) 837.

23. H. Zinnecker H. \& R. Mathieu, eds, IAU Symposium, The Formation of Binary Stars 2002001. 